\title{
Results of the verification of the NIR MOS EMIR
}

\author{
F. Garzón*a,b ${ }^{* a}$ N. Castro-Rodríguez ${ }^{\mathrm{a}}$, M. Insausti ${ }^{\mathrm{a}}$, L. López-Martín ${ }^{\mathrm{a}}$, Peter Hammersley ${ }^{\mathrm{b}}, \mathrm{M}$. \\ Barreto $^{a}$, P. Fernández ${ }^{a}$, E. Joven ${ }^{a}$, P. López ${ }^{a}$, A. Mato ${ }^{a}$, H. Moreno ${ }^{a}$, M. Núñez ${ }^{a}$ J. Patrón ${ }^{a}$, J.L.

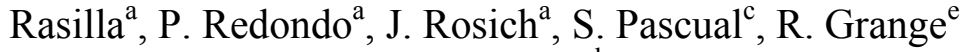 \\ ${ }^{a}$ Instituto de Astrofísica de Canarias, La Laguna, Spain; ${ }^{b}$ Departamento de Astrofísica, Universidad \\ de La Laguna, Spain; ${ }^{c}$ European Southern Observatory, Garching bei München, Germany; \\ dUniversidad Complutense, Madrid, Spain; 'Laboratoire d'Astrophysique de Marseille, Marseille,
}

France.

\begin{abstract}
EMIR is one of the first common user instruments for the GTC, the 10 meter telescope operating at the Roque de los Muchachos Observatory (La Palma, Canary Islands, Spain). EMIR is being built by a Consortium of Spanish and French institutes led by the Instituto de Astrofísica de Canarias (IAC). EMIR is primarily designed to be operated as a MOS in the $\mathrm{K}$ band, but offers a wide range of observing modes, including imaging and spectroscopy, both long slit and multiobject, in the wavelength range 0.9 to $2.5 \mu \mathrm{m}$. This contribution reports on the results achieved so far during the verification phase at the IAC prior to its shipment to the GTC for being commissioned, which is due by mid 2015. After a long period of design and fabrication, EMIR finally entered into its integration phase by mid 2013. Soon after this, the verification phase at the IAC was initiated aimed at configuring and tuning the EMIR functions, mostly the instrument control system, which includes a sophisticated on line data reduction pipeline, and demonstrating the fulfillment of the top level requirements. We have designed an ambitious verification plan structured along the three kind of detectors at hand: the MUX and the engineering and scientific grade arrays. The EMIR subsystems are being integrated as they are needed for the purposes of the verification plan. In the first stage, using the MUX, the full optical system, but with a single dispersive element out of the three which form the EMIR suite, the two large wheels mounting the filters and the pseudo-grisms, plus the detector translation unit holding the MUX, were mounted. This stage was mainly devoted to learn about the capabilities of the instrument, define different settings for its basic operation modes and test the accuracy, repeatability and reliability of the mechanisms. In the second stage, using the engineering Hawaii2 FPA, the full set of pseudo-grisms and band filters are mounted, which means that the instrument is fully assembled except for the cold slit unit, a robotic reconfigurable multislit mask system capable of forming multislit pattern of 55 different slitlets in the EMIR focal plane. This paper will briefly describe the principal units and features of the EMIR instrument as the main results of the verification performed so far are discussed. The development and fabrication of EMIR is funded by GRANTECAN and the Plan Nacional de Astronomía y Astrofísica (National Plan for Astronomy and Astrophysics, Spain).
\end{abstract}

Keywords: near-infrared instrumentation; multi-object spectroscopy; large ground based telescopes.

\section{INTRODUCTION}

The battery of $10 \mathrm{~m}$ class optical and near-infrared telescopes currently in operation, by sounding ever deeper into the Universe, hold the promise of providing, for the first time, a direct view of the processes that shaped the formation stars, galaxies and the Universe itself. Also, they are offering, again for the first time, the capability of detecting and isolating extragalactic stars and star forming regions with unprecedented sensitivity and resolving power, both spatial and spectral. A collective instrumentation effort in the late 80 's and is still underway to allow these new infrastructures to be used to their full potential. The scientific capabilities of the new telescopes are enormous, not only because of the larger photoncollecting area, but especially because of the new instruments, which, due to major technological advances, are orders of magnitude more efficient than their counterparts built decades ago for medium size collectors. In addition, these techno-

*fgl@iac.es; phone ++34 922605200; fax ++34 922605210; http://www.iac.es

Ground-based and Airborne Instrumentation for Astronomy V, edited by

Suzanne K. Ramsay, lan S. McLean, Hideki Takami, Proc. of SPIE Vol. 9147

91470U · (c) 2014 SPIE · CCC code: 0277-786X/14/\$18 - doi: 10.1117/12.2054804

Proc. of SPIE Vol. 9147 91470U-1 
logical challenges are establishing the first steps towards the construction of instrumentation for the forthcoming $30 \mathrm{~m}+$ class telescopes, now at the beginning of their design phases.

The Observatorio Roque de los Muchachos, operated by the Instituto de Astrofísica de Canarias (IAC) on the island of La Palma, is the site of the 10 meter Gran Telescopio Canarias (GTC) which started scientific operations in 2009. GTC is the largest aperture single dish telescope in world. Along this effort, a partnership of Spanish and French research institutions is working on the design and construction of EMIR, an advanced NIR multi-object spectrograph for GTC, which will be visited in this paper.

EMIR (Espectrógrafo Multi-objeto InfraRrojo) ${ }^{1,2,3,5}$ is a common-user, wide-field camera-spectrograph operating in the near-infrared (NIR) wavelengths 0.9-2.5 $\mu \mathrm{m}$, using cryogenic multi-slit masks as field selectors. As a reminder, its main instrumental features and expected capabilities, in terms of sensitivities in the two observing modes, are given in table 1. EMIR will provide GTC with imaging, long-slit and multi-object spectroscopic capabilities. The EMIR consortium is formed by the IAC, Universidad Complutense de Madrid (UCM, Spain), the Laboratoire d'Astrophysique de ToulouseTarbes (LATT, France) and the Laboratoire d'Astrophysique de Marseille (LAM, France). Specifications are listed in table 1. EMIR will provide GTC with imaging, long-slit and multi-object spectroscopic capabilities). EMIR is now at the middle of its System Verification Phase, and is due for first commissioning at the GTC in mid 2015. This phase is being funded by GRANTECAN and the Plan Nacional de Astronomía y Astrofísica.

Table 1 Top level specifications of EMIR.

\begin{tabular}{|c|c|}
\hline Wavelength range & $0.9-2.5 \mu \mathrm{m}$ \\
\hline Optimization & $1.0-2.5 \mu \mathrm{m}$ \\
\hline Observing modes & $\begin{array}{l}\text { Multi-object spectroscopy } \\
\text { Wide-field Imaging }\end{array}$ \\
\hline Top priority mode & K band Multi-object spectroscopy \\
\hline Spectral resolution & $5000,4250,4000(\mathrm{JHK})$ for $0.6 "$ (3-pixel) wide apertures \\
\hline Spectral coverage & One observing window $(\mathrm{Z}, \mathrm{J}, \mathrm{H}$ or $\mathrm{K})$ per single exposure \\
\hline Array format & 2048x2048 HgCdTe (Rockwell-Hawaii2) \\
\hline Scale at detector & $0.2 \operatorname{arcsec} /$ pixel \\
\hline Image quality & $\theta_{80}<0.3 \operatorname{arcsec}$ \\
\hline \multicolumn{2}{|c|}{ Multi-object spectroscopic mode } \\
\hline Slit area & $\begin{array}{l}6,7 \times 4 \text { arcmin, with } 55 \text { slitlets of } \sim 7 " \text { long and width } \\
\text { varying between } 0.4 \text { and } 1 \text { arcsec }\end{array}$ \\
\hline Sensitivity & $\begin{array}{l}\mathrm{K}<20.1, \mathrm{t}=2 \mathrm{hrs}, \mathrm{S} / \mathrm{N}=5 \text { per } \mathrm{FWHM} \text { (continuum) } \\
\mathrm{F}>1.4 \times 10^{-18} \mathrm{erg}^{-1} \mathrm{~s}^{-1} \mathrm{~cm}^{-1} \AA^{-1}, \mathrm{t}=4 \mathrm{hr}, \mathrm{S} / \mathrm{N}=5 \text { per FWHM (line) } \\
\quad \text { Image mode }\end{array}$ \\
\hline FOV & $6,7 \times 6,6 \operatorname{arcmin}$ \\
\hline Sensitivity & $\mathrm{K}<22.8, \mathrm{t}=1 \mathrm{hr}, \mathrm{S} / \mathrm{N}=5$, in 0.6 " aperture \\
\hline
\end{tabular}

\section{OPTOMECHANICAL LAYOUT}

The optical and mechanical concepts of EMIR have been studied from many approaches in order to have a good balance between the performance of the instrument, the technical risks and the global price. The EMIR requirements make the concept extremely challenging, and the design approaches have tried to minimize the trade off between requirements and technical solutions.

The optical train ${ }^{6,7,8}$, all in transmission, is composed from end to end by a cryostat window, acting as a field lens and powered for flattening the GTC focal surface, where the Cold Mask Unit (CSU) $)^{9,10,11}$ is located. Then a multiple spherical lens collimator, combining a single lens and a triplet forms the image of the GTC secondary at the pupil plane, where the dispersive elements and Lyot stop can be inserted and removed from the beam with a rotary wheel. A six element camera, all of them spherical except the last one, focus the beam onto the detector after crossing the filter wheel 
situated between the last camera lens and the detector, mounted on a XYZ motion table, the Detector Translation Unit $(\mathrm{DTU})^{12}$. All lenses, including the field lens are AR coated in the two surfaces.

The EMIR mechanical design ${ }^{13,14,15,16}$ relays on the development of a fully cryogenic robotic system which can be remotely reconfigured to form the multi-slit pattern in the instrument focal plane, the CSU, which has been subject to a development and manufacturing contract and is pending of its final acceptance at the IAC, which should happen in the forthcoming weeks. Several functional prototypes have been produced along during the development phase, which has been tested intensively to optimize the concept.

Two other mechanisms have been developed in the course of the project: the wheels for the grisms and filters, which shares a common concept; and the DTU, an XYZ motorized positioning bench for the detector. The continuous use of the DTU will permit active compensation of the internal flexures of the instrument, attached to the Nasmyth rotator and will also be used to implement advanced features in the observing strategy

\section{CONCEPT OF THE SYSTEM VERIFICATION PHASE}

The system level AIV EMIR is based around cool downs of the instrument cryostat. The first cool down is to demonstrate the operation of the main mechanical units (except the CSU) at the operational temperatures and in situ then in each succeeding cycle more capability is added until on the last cool down the final acceptance testing of the instrument is done. Each cool down has clearly defined goals and if these are not satisfactorily demonstrated the cool down is repeated.

For this to work it is assumed that the individual components have already been tested and what is being done here is to integrated the component into the instrument and then characterizes its performance in situ.

The AIV makes use of the 3 types of detector that are at our disposal: the multiplexor, the engineering array and the science grade array, with the aim maximising efficiency and of having the systems and procedures sufficiently well tested and safe so as to minimise the risk of damage to the science grade array.

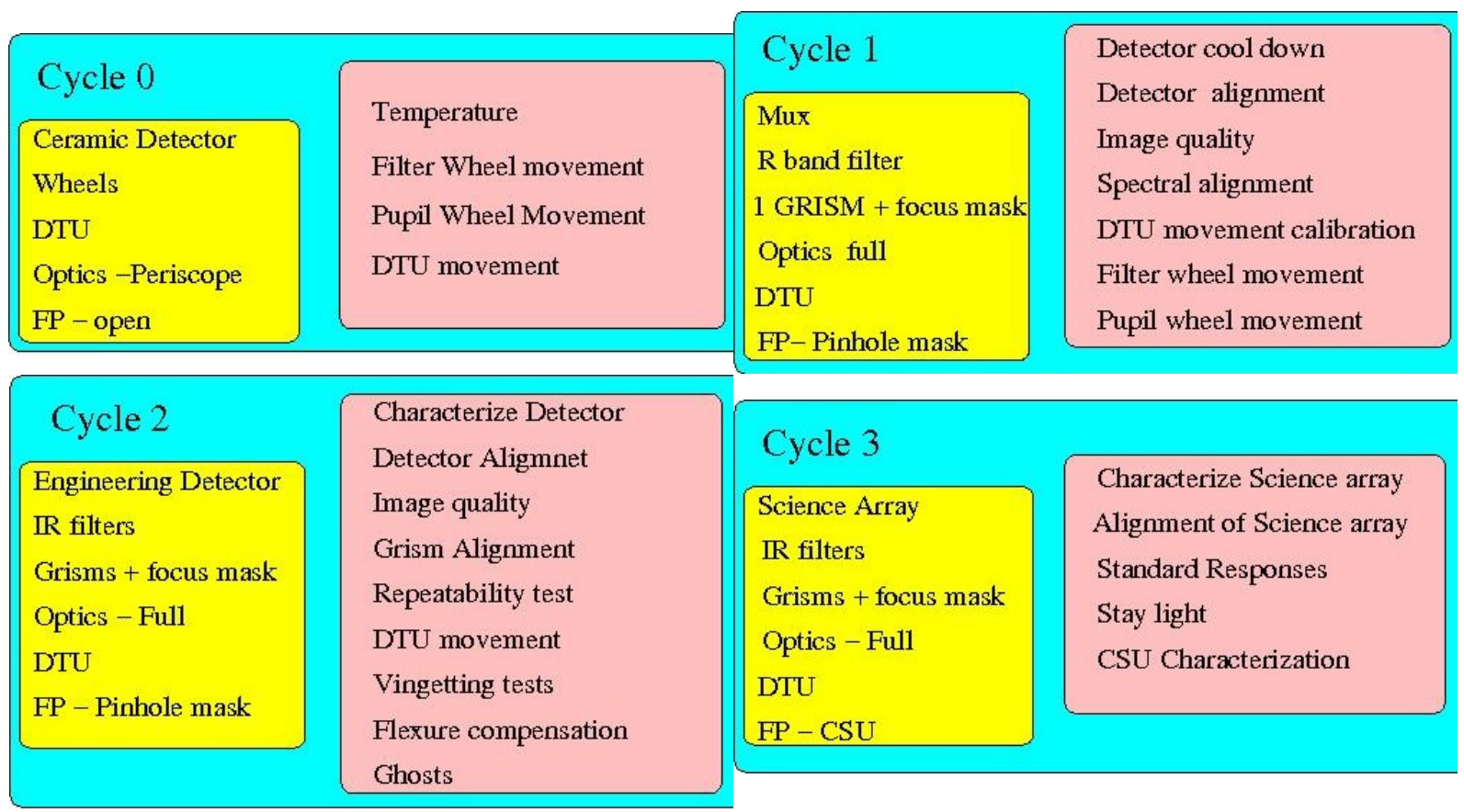

Figure 1. Scheme of the different stages of the system verification.

As the CSU is not planned to be available until the last cycle, a pinhole mask is in place in the focal plane for the earlier cycles. This pinhole mask has two regular pinhole patterns, whose images are roughly $1 \mathrm{px}$ and $3 \mathrm{px}$ in diameter at the detector, plus several slits and 4 big openings on each detector quadrant to measure sensitivity against different illumination fluxes and uniformity pixel to pixel, which is important for flat-fielding. The geometry and location of the features of the mask have been measured with accuracies better than 10 microns, figure that has to be aligned with the 
projected pixel size at the focal plane of 166 microns. So the pinhole mask is known with an accuracy of around 1/16 of the pixel size. Figure 1 gives an overview of the process.

At the time of this writing, stages 0 and 1 have been fully covered, with their goals and aims sufficiently achieved so has very little has been left behind for the next stage. We have just finished the first cooldown of stage 2, composed by two nominal cooldowns, on which the pinhole mask has been in place and the geometry of the EMIR focal plane has been surveyed. The second and last cooldown of this stage will not mount the mask, so as the illumination of the pupil can be tested. It is expected that in the final stage, with the science detector, EMIR will be equipped with the CSU, which will then permit to place different illumination pattern as well as full image mode in the focal plane. Hence, the full set of objectives can be covered in a single cooldown, if successful.

In short, the main objectives of the System level AIV can be grouped into one or several of the following categories:

- To work out the operation procedures of the cryostat: pre cool down, cool down, warm up, etc.

- To optimise the detector operations, run up the Detector Acquisition System (DAS) and work out and optimize the read-out modes. Measure read noise levels, interferences between mechanisms and detector, etc.

- Measure image quality and related parameters, over the full FOV and wavelength regime.

- To measure and correct the tilt between the detector and the DTU, and between the DTU and the focal plane.

- To commission the repeatability of verification procedures and perform full repeatability tests.

- Flexure tests and measure of the impact of flexures on the instrument performances. Establish DTU compensation.

- To measure and correct any tilt between the detector and the focal plane.

- Stray light test

- To operate the CSU in situ and perform full checking, including the calibration procedures.

- Operate the instrument control system in place. Establish and optimize the connection with the on-line Data Reduction Pipeline (DRP).

In the remaining of this paper, some recent results achieved so far in several of the above categories will be reported. It is of some interest to note that the full system AIV is being performed with EMIR installed onto its integration tool, while the instrument has been also mounted on the Nasmyth rotator machine at the IAC integration room to check the interfaces with the GTC.

\subsection{The control system}

A very important part of the AIV process is being the integration and debugging of the control system. EMIR has to follow the GTC standard for the control software ${ }^{17}$ (GCS) which imposes severe restrictions to the development. From the standpoint of the GCS, the EMIR control software ${ }^{18,19}$ (ECS) can be regarded as a collection of components that integrate into the GCS and are interconnected among them and with the rest of the GCS, largely through a CORBA manager. Since the start of the system verification, a major task, which surpasses the rest of the test plan, is being the specification, debugging and optimization of the ECS, which must coordinate all the EMIR operations.

In addition, EMIR has its own Data Reduction Pipeline ${ }^{20,21,22,23}$ (DRP), which is a key component of the instrument. Being a NIR MOS and imager, most of the EMIR observations will be split in large sequences of short integration time each, which must be treated properly, and in real time, to permit a correct assessment of the quality control. This software component is not developed within the GCS but communicates smoothly to it, in both directions. Again, the tuning of this software, composed by a large set of reduction recipes, constitutes a major task of the system verification effort.

During the verification, the ECS and DRP are being treated simultaneously as verification tools and as EMIR subsystem which need to be tested. These are the only two subsystems that are being verified as such during this phase.

\section{SOME RESULTS OF THE VERIFICATION}

\subsection{Detector and DTU alignment in the EMIR image plane.}

This is an iterative process on which the results on previous cooldowns serve as feedback for the next ones. The detector is sited on its assembly with a gimbal type mount, thus permitting its tilt over three fix axis, one of this should coincide with the optical axis, Z, being the other two in plane perpendicular to it. With the use of the MUX detector, sensitive to 
the optical bands and functional at room temperature (RT), the array is first aligned at RT. To this end, the centroid of the pinhole images are determined and a two stages plane fitting is made to the series of the pinhole positions varying the $\mathrm{Z}$ position of the array with the use of the DTU. Acting on the tilting screws of the detector assembly, when at RT, the best position of the full system is then selected. Typical residual tilt angles of the detector are of the order of 1-2 mrad.

At cold, the process is then repeated, this time without realignment, and the shift in detector tilt angles with respect to the result at RT is taken as an input for the next cooldown. Previous to all these processes, the metrology of the pinhole mask have been measured once mounted into EMIR to get the position of each feature with respect to the instrument coordinate system, thus getting the position of the instrument optical axis. Then, the location of the optical axis on the detector with the DTU centred or, alternatively, the position of the DTU which centres the detector on the field, are obtained. In the last cooldown, at cryo, those positions result to be:

- Mask position with respect to EMIR: tilt: $0,03^{\circ} ; \mathrm{X}$-shift=0,570mm; Y-shift=-0.456mm

- Position of the optical axis on image plane:

○ Detector (DTU at $(0,0)):$ X0[pxs]: 0.698; Y0[pxs]: -25.375

○ DTU: XDTU0[microns]: 469.150; YDTU0[microns]: 13.082

It is to note that the DTU XY axes are rotated $90^{\circ}$ with respect of those of the detector. Also the position of the detector in the image plane is not optimal and will be corrected before the next cooldown. The residual tilt of the detector in image plane is then computed on each filter and in 12 rotation position angle of the instrument (IPA), together with the best focus, given consistent results. As an example, for the Brackett- $\gamma$ narrow band filter at IPA $=0$, one gets:

- $\quad$ Best Z (microns): $572.293+/-11.326$

- $\quad$ Tilt over X (horizontal, mrad): $2.361+/-0.461$

- Tilt over Y (vertical, mrad): $1.587+/-0.441$
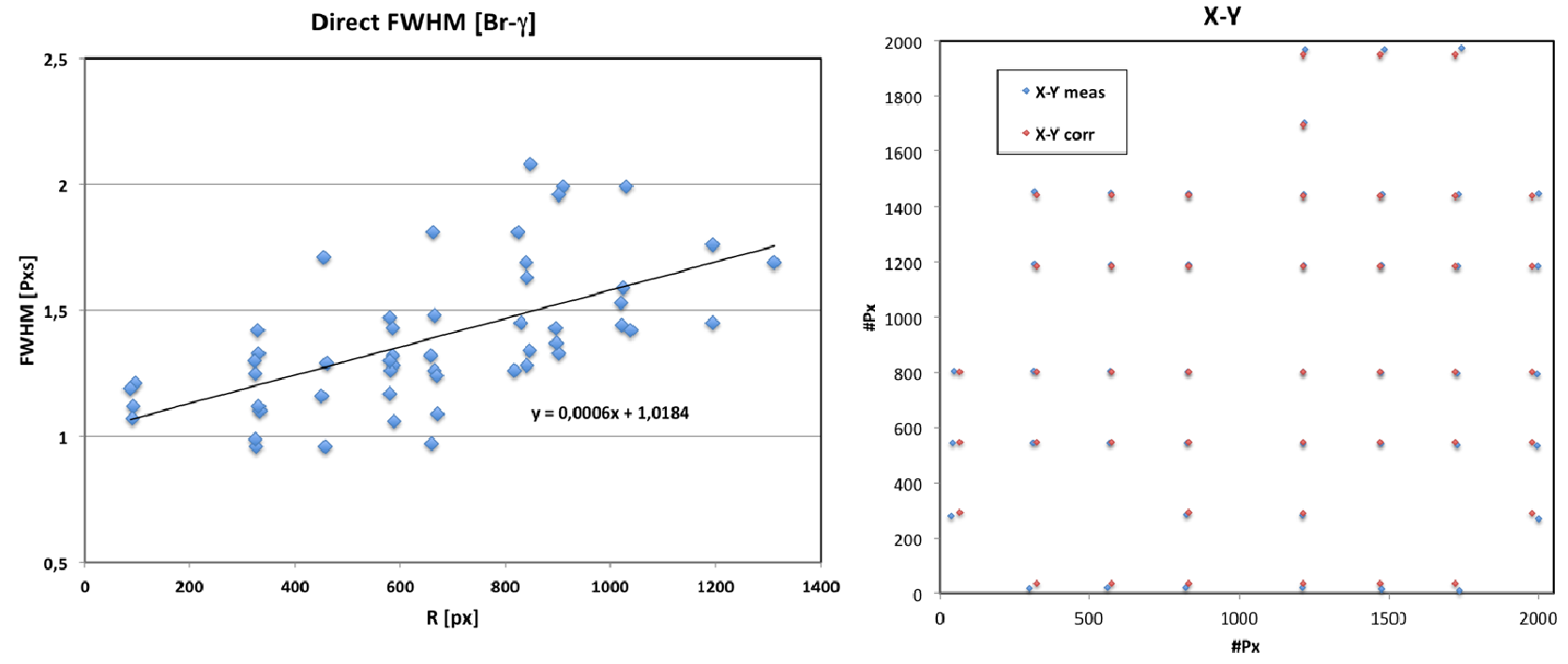

Figure 2. Left panel: direct measurement of the FWHM of the pinhole images represented versus radial distance from the detector centre. Right panel: geometrical distortion of the pinhole images shown with the double mark per pinhole: the measured and the corrected one, using the position of the pinhole in the mask and the nominal plate scale.

\subsection{Image quality and optical distorsion.}

With the use of the pinhole mask, the PSF distribution of the images of the pinholes has been measured for the full set of broad-band filters $\left(\mathrm{YJHK}_{\mathrm{s}} \mathrm{KK}_{\text {spec }}\right)$ and narrow band filters $(\mathrm{Fe}-\mathrm{II}+\mathrm{Br}-\gamma)$ which equip the instrument at present. The results are very much in line with the expectations and well within specs.

For PSF measurements, we have used the pinholes of $1 \mathrm{px}$ width, which are more appropriate for image quality tests, while the 3px wide pinholes have been selected for determining centroids and hence optical distortion. In numbers, and for the Br- $\gamma$ narrow band filter, the average PSF is $1,39 \pm 0,29 \mathrm{pxs}$; and the geometrical distortion mean value is $0.89 \%$ $+/-0.018$ over the whole FOV. In figure 2, the details of these numbers are presented. 


\subsection{Preliminary stray light measurements.}

While a proper estimate of the unwanted light a the EMIR detector cannot be achieved with the pinhole mask blocking most of the focal plane, some insight of the amount of diffuse and/or stray light can be gained by flashing the instrument with a source of varying power and look at the detector signal in regions outside, but close to, the images of the openings.

Some results are displayed in figure 3 , where it can be seen that the regions outside the opening increase the signal by an amount from 50 to 100 counts from the pedestal level when the signal at the opening is at mid point before saturation.
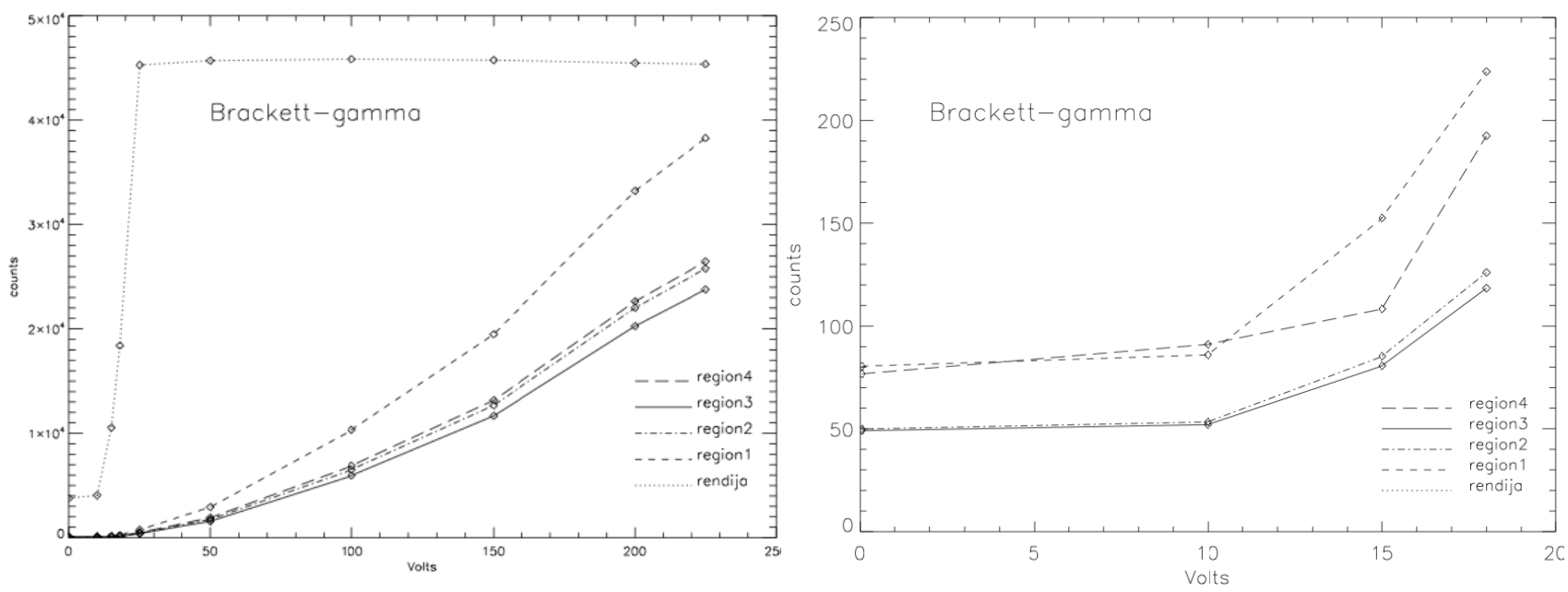

Figure 3. Mean detector signal on regions distributed over the FOV, at varying distances from the opening image. Regions are typically $100 * 100$ pixels in area and include a pinhole image. The abscissae give the input voltage of the illuminating source. Left panel: full illumination range. Right panel: zoom up to the voltage on which the signal at the opening is at mid well depth.

\subsection{Read-out modes.}

EMIR offers a wide range of read-out modes, which has been described elsewhere ${ }^{24,25}$. It is an important goal of the instrument performances that all these modes are photometrically compatible among them, so as to ease the astronomical calibration procedures when in telescope. In figure 4, some results are shown, over two detector quadrants, which proves the compatibility over the full well depth of the detector.

Counts_1

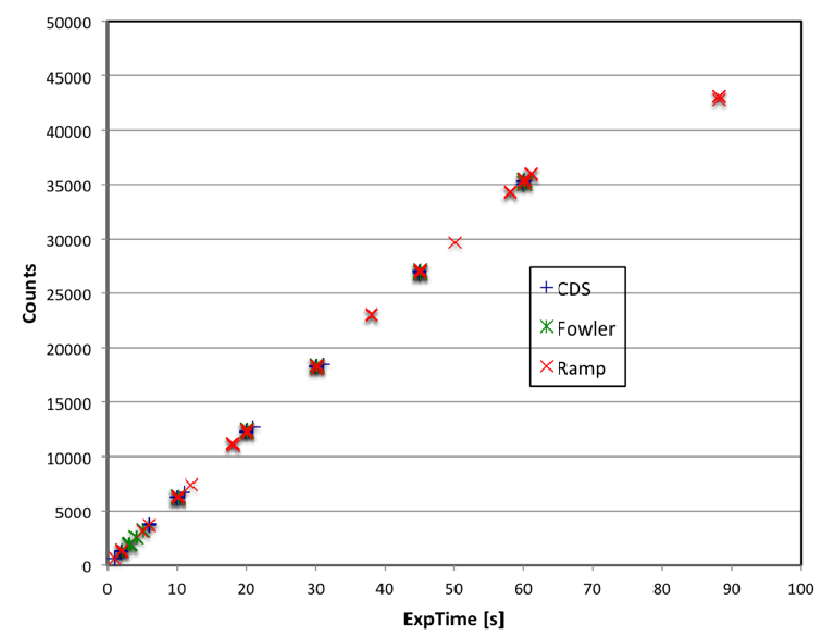

Counts_3

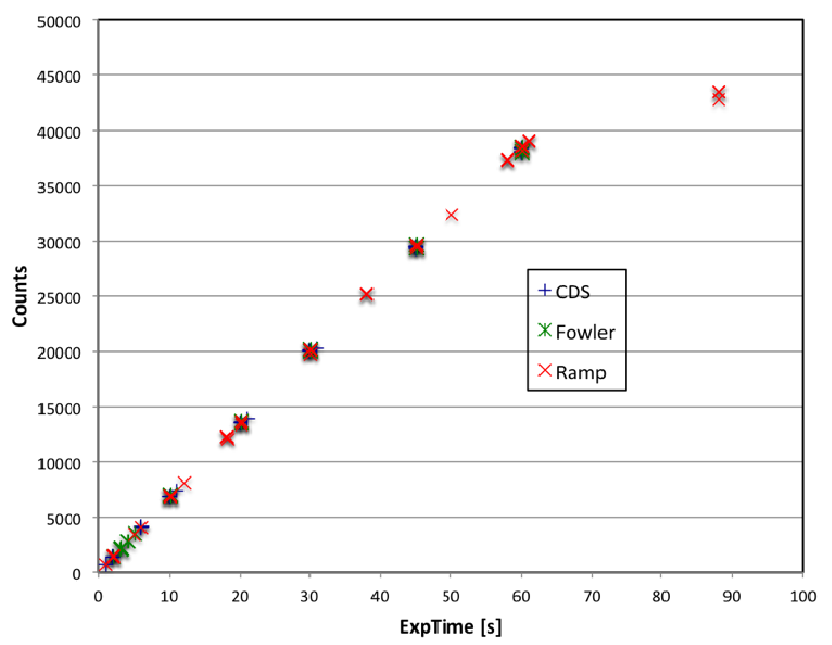

Figure 4. Signal vs. exposure time for an illumination of constant power source, taken as averages over sufficiently big areas on each of the openings, and using different readout modes. 
4.5 On instrument DTU performances.
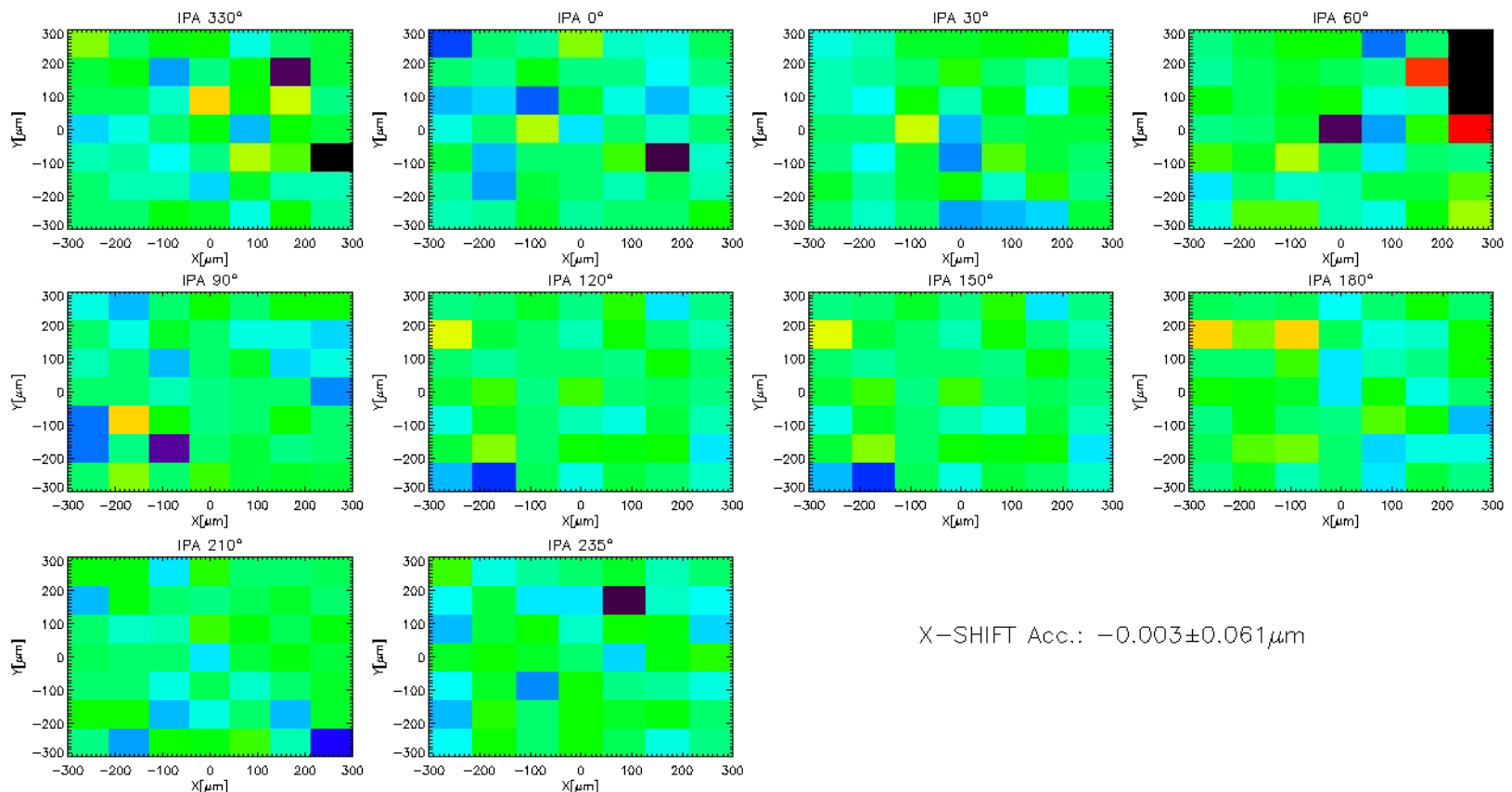

X-SHIFT Acc.: $-0.003 \pm 0.061 \mu \mathrm{m}$

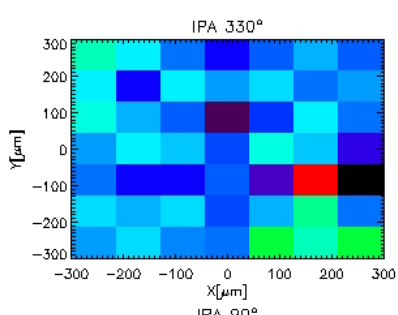

$-0.2$
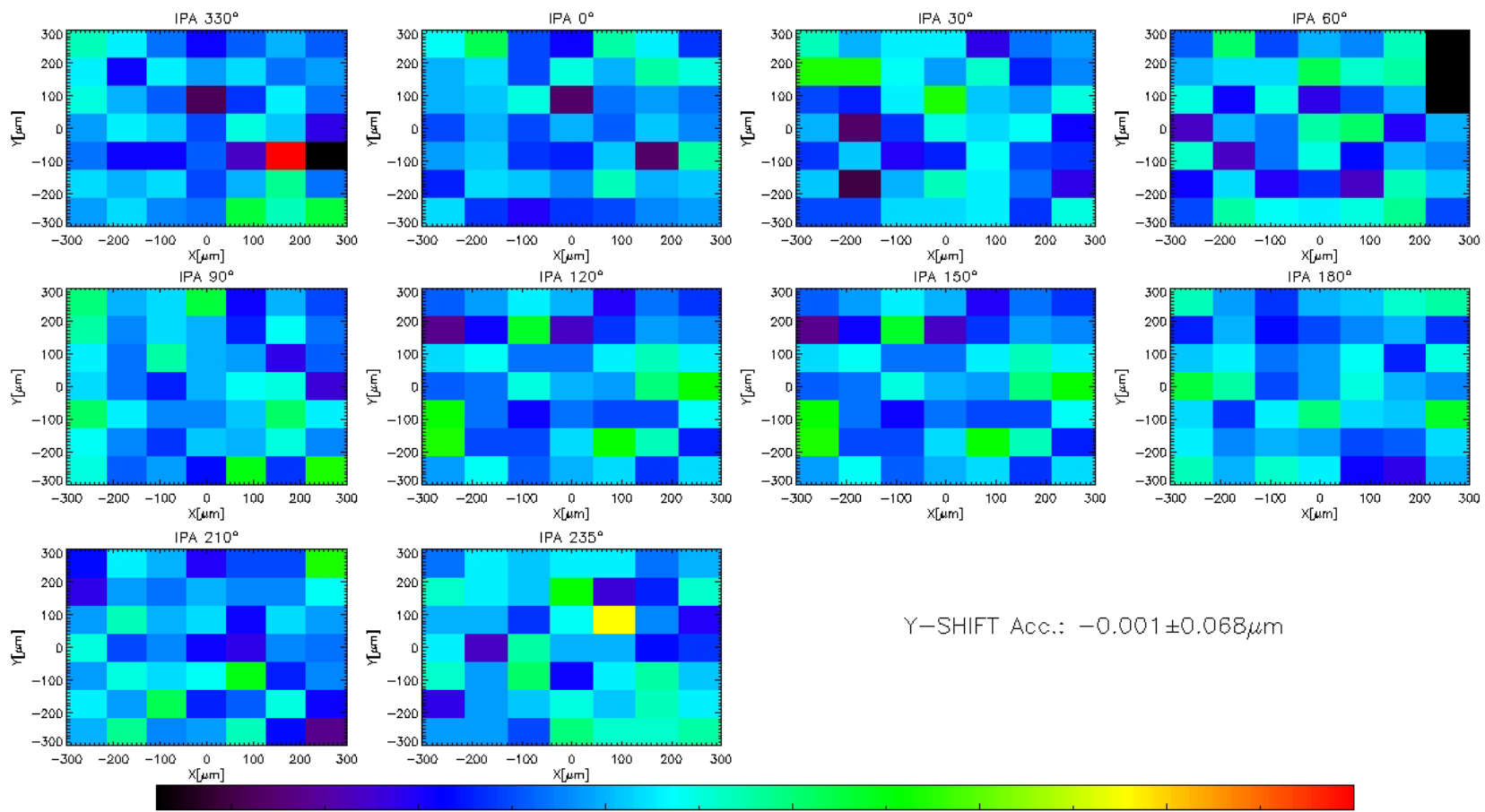

$-0.1$

Y-SHIFT Acc:: $-0.001 \pm 0.068 \mu \mathrm{m}$

Figure 5. Accuracy of the DTU motion in X (top panel) and Y (bottom panel), in microns, over the full set of IPAs that the integration tool can cover.

The DTU is an extremely importat subsystem in the EMIR instrument as it has to correct the internal flexures while the instrument is rotating at the Nasmyth station during integrations. It has to be reminded that it is anticipated that EMIR will be mostly used with long integration times in weak sources. As well as debugging and optimizing the control 
software component of this unit and integrating it at the ECS, the verification tests on this component have been basically of two types. First, checking its functionality, once integrated and mounting the detector on it, under varying instrument position angle in the integration tool. In the integration tool EMIR cannot perform a full rotation but can cover most of the turn.

On each IPA, a long series of DTU XY motions are commanded over most of the DTU XY reach, and the accuracy, shown in figure 5, and repeatability are computed by comparing the position of the DTU given by its sensors and the centroids of the pinhole images on each DTU position. As can be seen, the results are excellent.

\subsection{DTU flexure compensation.}

Once the previous tests have been completed, one can proceed to the first stage of the DTU flexure compensation procedure. Using the recorded positions, a first draft of the XY shift lookup table is built by fitting over the 12 IPAs measured. The fit provides the DTU compensation every degree. Then a complex test was done on which the integration tool rotates at a fixed angular speed of 1 degree every 6 seconds and the images were acquired continuously and being reduced on-line by the DRP. At the end, the pattern of the displacement due to internal flexures can be established.
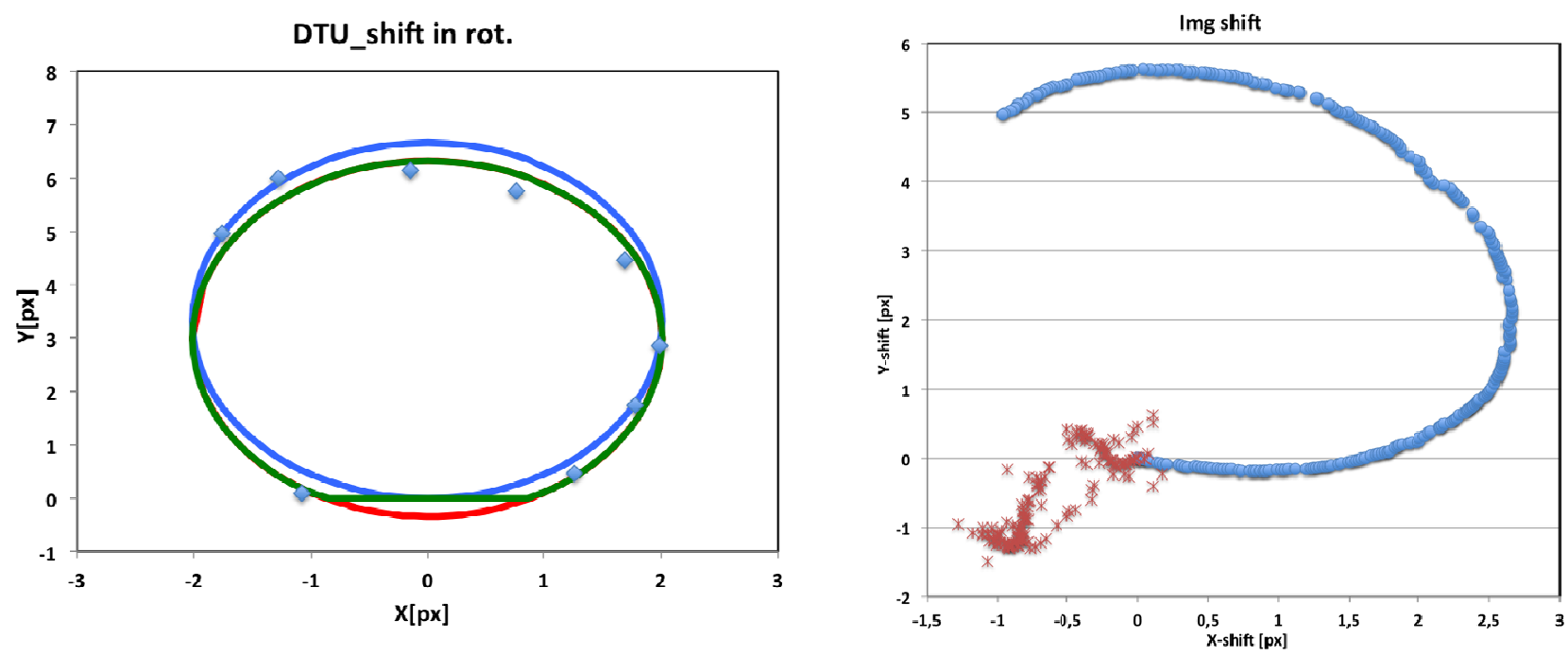

Figure 6. Left panel: several fits of the XY shifts over the measured range of IPAs. Right panel: averaged displacement of the pinhole images when rotating EMIR over the permitted range of the integration tool. Filled circles show the averaged shift of the images without DTU correction, and the crosses, with the DTU correction in place.

As can be seeing in figure 6, the DTU is fully capable to keep the detector at a fix position with respect to the sky at all times, while the lookup table used in this test lacks sufficient accuracy to reproduce the shifts. These results will be used to refine further the lookup table that will be tested again in the next verification stage.

\subsection{Calendar to the first light.}

As mentioned in the abstract, EMIR is due for commissioning by mid 2015. The tasks that the EMIR team has to face until that time can be summarized as follows:

- Complete system AIV stage 2. The second cooldown, without the pinhole mask, is planned to start next July 2014. Prior to it, some corrective actions on the instrument must be completed.

- Accept the CSU unit and finalize its control software component. The acceptance tests commencement are planed for the next weeks.

- Complete system AIV stage 3, with the full instrument integrated, thus the CSU.

- Execute the acceptance test plan previously agreed with the GTC project office. A first version of the plan has been already discussed. The second and, hopefully, final version is on the way.

- Ship the instrument to the observatory and install it at the GTC Nasmyth station to initiate the commissioning. The commissioning plan has to be already agreed between the parties with sufficient time prior to this action. As before, a first version of this plan has been already circulated. 


\section{ACKNOWLEDGEMENTS}

This work was performed at the Instituto de Astrofísica de Canarias as part of the development of the EMIR instrument to be installed at the GTC telescope. EMIR is supported by the Spanish Ministry of Economy and Competitiveness (MINECO) under the grant (project reference AYA2012-33211), GRANTECAN, S.A., via a dedicated contract, and the EMIR partners institutions, in alphabetical order, IAC, LAM, LATT and UCM.

\section{REFERENCES}

[1] Garzón, F., Abreu, D., Barrera, S., Becerril, S., Cairós, L. M., Díaz, J. J., Fragoso, A. B., Gago, F., Grange, R., González, C., López, P., Patrón, J., Pérez, J., Rasilla, J. L., Redondo, P., Restrepo, R., Saavedra, P., Sánchez, V., Tenegi, F., and Vallbé, M., "EMIR, the GTC NIR multi-object imager-spectrograph", Rev. Mex. A\&A Conf. Series 29, 12-17 (2007).

[2] Garzón, F., Abreu, D., Barrera, S., Becerril, S., Cairós, L. M., Díaz, J. J., Fragoso-López, A. B., Gago, F., Grange, R., González, C., López, P., Patrón, J., Pérez, J., Rasilla, J. L., Redondo, P., Restrepo, R., Sánchez, V., Tenegi, F., and Vallbé, M., "EMIR, the GTC NIR Multiobject Imager-Spectrograph", Highlights of Spanish Astrophysics IV 81- (2007).

[3] Garzón, F., Abreu, D., Barrera, S., Becerril, S., Cairós, L. M., Díaz, J. J., Fragoso, A. B., Gago, F., Grange, R., González, C., López, P., Patrón, J., Pérez, J., Rasilla, J. L., Redondo, P., Restrepo, R., Saavedra, P., Sánchez, V., Tenegi, F., and Vallbé, M., "EMIR: the GTC NIR multi-object imager-spectrograph", Proc. SPIE 6269 (2006).

[4] Garzón, F., Abreu, D., Barrera, S., Correa, S., Díaz, J. J., Fragoso, A. B., Fuentes, F. J., Gago, F., Gonzalez, C., López, P., Manescau, A., Patrón, J., Pérez, J., Redondo, P., Restrepo, R., Sanchez, V., and Villegas, A., "EMIR: the GTC NIR multi-object imager-spectrograph", Proc. SPIE 5492, 1187-1195 (2004).

[5] Garzón, F., Barrera, S., Correa, S., Díaz -García, J. J., Fragoso-López, A. B., Fuentes, F. J., Gago, F., LópezRuiz, J.-C., Manescau, A., Patrón, J., Pérez-Espinos, J., Sanchez de la Rosa, V., Villegas, A., and Redondo, P., "EMIR: the GTC NIR multiobject imager-spectrograph", Proc. SPIE 4841, 1539-1547 (2003).

[6] Manescau, A., Fragoso-López, A. B., Garzón, F., and Patrón, J., "Status report on the EMIR optical system", Proc. SPIE 5492, 1735-1742 (2004).

[7] Manescau, A., Fragoso-López, A. B., Garzón, F., and Patrón, J., "Status of the EMIR optical system", Proc. SPIE 4841, 230-239 (2003).

[8] Manescau, A., Fragoso-López, A. B., Balcells, M., Ballester Lluch, J. A., Correa, S., Díaz-García, J. J., Fuentes, F. J., Gago, F., Garzón, F., López-Ruiz, J. C., Patrón, J., and Pérez-Espinos, J., "The EMIR optical system", Proc. SPIE 4411, 39-46 (2002).

[9] Teuwen, M., Janssen, H., Casalta, J. M., and Garzón López, F., "Integration status of the configurable slit unit for GTC-EMIR", Proc. SPIE 8446 (2012).

[10] Teuwen, M., Janssen, H., Geurink, R., Boots, M., and Garzón López, F., "GTC-EMIR configurable slit unit development details", Proc. SPIE 7018 (2008).

[11] Teuwen, M., Janssen, H., Geurink, R., Redondo, P., and Díaz-García, J. J., "Development of configurable slit unit for GTC-EMIR", Proc. SPIE 6269 (2006).

[12]Zago, L., Droz, S., Racz, L., and Molins, A., "The EMIR detector translation unit: a cryogenic high-precision 3DoF parallel mechanism", Proc. SPIE 6273 (2006).

[13] Sánchez, V., Barrera, S., Becerril, S., Correa, S., Pérez, J., Redondo, P., Restrepo, R., Saavedra, P., Tenegi, F., Patrón, J., and Garzón, F., "Status of the EMIR mechanical system", Proc. SPIE 6269 (2006).

[14] Barrera, S., Villegas, A., Fuentes, J., Correa, S., Pérez, J., Redondo, P., Restrepo, R., Sánchez, V., Tenegi, F., Garzón, F., and Patrón, J., "EMIR optomechanics", Proc. SPIE 5495, 611 (2004).

[15] Correa, S., Restrepo, R., Tenegi, F., Fuentes, F. J., Sanchez, V., Barrera, S., Pérez, J., Redondo, P., Villegas, A., Garzón, F., and Patrón, J., "Structural and thermal analysis of EMIR", Proc. SPIE 5492, 1331-1342 (2004).

[16]Fuentes, F. J., Sanchez, V., Barrera, S., Correa, S., Pérez, J., Redondo, P., Restrepo, R., Tenegi, F., Villegas, A., Patrón, J., and Garzón, F., "EMIR mechanical design status", Proc. SPIE 5492, 1319-1330 (2004).

[17] Gómez-Álvarez, P., García-Dabó;, C. E., Osinde, J., and Macíacute;as, R., "The GTC Telescope Control and Data Reduction Systems", Proc. SPIE 351, 559- (2006) 
[18] López-Ruiz, J. C., Joven, E., López, P., Díaz , J. J., Aguiar, M., Herrera, A., Gigante, J. V., Cepa, J., Garzón, F., Patrón, J., and Pérez, A., "EMIR and OSIRIS instruments: common data acquisition software architecture", Proc. SPIE 5496, 438-445 (2004).

[19] López-Ruiz, J. C., Díaz, J. J., Gago, F., Garzón, F., Patrón, J., Pelló, R., and Gallego, J., "EMIR software: an overview", Proc. SPIE 4848, 474 (2002).

[20] Pascual, S., Gallego, J., Cardiel, N., and Eliche-Moral, M. C., "PyEmir: Data Reduction Pipeline for EMIR, the GTC Near-IR Multi-Object Spectrograph", Proc. SPIE 434, 353- (2010).

[21] Pascual, S., Gallego, J., and Cardiel, N., "A Fully GTC-Compliant Pipeline for the Direct Imaging Mode of EMIR", Highlights of Spanish Astrophysics V 523- (2010).

[22] Pascual, S., Gallego, J., and Cardiel, N., "Data Reduction Pipeline for EMIR, the Near-IR Multi-Object Spectrograph for GTC", Rev. Mex. A\&A Conf. Series 29, 142-142 (2007).

[23] Pascual, S., Gallego, J., Cardiel, N., Zamorano, J., Gorgas, F. J., García-Dabó, C. E., and Gil de Paz, A., "Data Reduction Pipeline for EMIR, the Near-IR Multi-Object Spectrograph for GTC", Proc. SPIE 351, 279- (2006).

[24] Núñez, M., Gago, F., Garzón, F., Díaz, J. J., Barreto, M., Patrón, J., González-Fenández, C., Hammersley, P. L., López, L., and Castro, N., "EMIR high-dynamic range readout modes", Proc. SPIE 8453 (2012).

[25] Díaz, J. J., Gago, F., López, P., Beigbeder, F., Garzón, F., and Patrón, J., "EMIR Detector Data Acquisition Electronics", Scientific Detectors for Astronomy 2005 627- (2006). 